\title{
Nonlinear Strain Theory of Plastic Flow in Solids
}

\author{
Akira Onuki \\ Department of Physics, Kyoto University, Kyoto 606-8502, Japan
}

\begin{abstract}
We present a phenomenological time-dependent Ginzburg-Landau theory of nonlinear plastic deformations in solids. Because the problem is very complex, we first give models in one and two dimensions without vacancies and interstitials, where large strains produce densely distributed slips but the mass density deviations remain small except near the tips of slips. Next we set up a two-dimensional model including a vacancy field (or local free-volume fraction), where relevant is the sensitive dependence of the elastic shear modulus on the vacancy density. In our simulation, if strains are applied to nearly defectless solids but in the presence of such elastic inhomogeneity, the vacancy density and the mass density can become considerably heterogeneous for large strains on spatial scales much longer than the atomic size. These strain-induced disordered states are metastable or long-lived once they are created.
\end{abstract}

\section{Introduction}

Most previous papers on glass transitions so far are concerned with near-equilibrium properties such as relaxations of the density time correlation functions or dielectric response. However, these quantities are too restricted or indirect, and there remains a rich group of unexplored problems in far-from-equilibrium states. In particular, shear is a relevant perturbation drastically changing the glassy dynamics when the shear rate $\dot{\gamma}$ exceeds the inverse of the structural relaxation time $\tau_{\alpha}[1,2]$. In supercooled liquids, the microscopic rearrangement processes occur on the time scale of $\tau_{\alpha}$ in quiescent states, whereas they are much accelerated even by extremely small $\dot{\gamma}$ (if larger than $\tau_{\alpha}^{-1}$ ). Similar jamming rheology has been studied in systems composed of large elements such as colloidal suspensions, dense microemulsions, and granular materials [3].

Experimentally, Simmons et al. [4] observed shear-thinning behavior, where the steady-state viscosity $\eta(\dot{\gamma})$ was represented by

$$
\eta(\dot{\gamma})=\sigma_{x y} / \dot{\gamma} \cong \eta(0) /\left(1+\dot{\gamma} \tau_{\eta}\right),
$$

in the range $7 \times 10^{5}<\eta(0)<6 \times 10^{13}$ (Poise) in soda-lime-silica glass. The characteristic time $\tau_{\eta}$ is expected to be of order $\tau_{\alpha}$. Remarkably, the shear stress $\sigma_{x y}$ tends to a limiting shear stress, $\sigma_{\lim }=\eta(0) / \tau_{\eta}$, of order $10^{-2} \mu_{0}, \mu_{0}$ being the shear modulus for infinitesimal 
strains. After application of shear, they also observed an overshoot of the shear stress before approach to a steady state.

As a closely related problem, understanding of mechanical properties of amorphous metals has been of great technological importance $[5,6]$. They are usually ductile in spite of their high strength. At low temperatures $T \lesssim 0.6 \sim 0.7 T_{g}$, localized shear bands, where zonal slip occurs, have been observed above a yield stress. At relatively high temperatures $T \gtrsim 0.6 \sim 0.7 T_{g}$, on the other hand, shear deformations are induced homogeneously (on macroscopic scales) throughout samples, giving rise to viscous flow with strong shear thinning behavior. In a model amorphous metal in 3D, Takeuchi et al. [7] numerically followed atomic motions after application of a small shear strain to observe heterogeneities among poorly and closely packed regions. Such dynamic heterogeneities have been reported in recent simulations in sheared states [1] and also in quiescent states $[8,9,10,11]$.

In the study of elasto-plastic dynamics of solids, microscopic simulations are informative $[12,13,14]$, while mean-field theories are instructive $[15,16]$. In the latter theories, the problem was reduced to to that of one element obeying a stochastic process under the influence of the average stress. The aim of this paper is then to present a spacetime dependent, elasto-plastic dynamical model on the basis of well-defined nonlinear elastic theory. Although our theory is still preliminary, we shall see that introduction of an order parameter, representing the vacancy or the local free volume, can give rise to dramatic effects similar to those reported in Ref.[12].

\section{Plastic flow in one dimension}

To introduce the fundamental concepts in our problem in the simplest manner, we first present a one-dimensional model which mimics a solid with shear deformations varying only in one direction (1D slip model). We here write down the dynamic equations in the continuum representation (for simplicity). The velocity $v(x, t)=\partial u(x, t) / \partial t$ of the shear displacement $u(x, t)$ (along the $y$ axis) is governed by

$$
\rho \frac{\partial}{\partial t} v=\nabla_{x}\left[\mu_{0} \gamma_{0} \sin \left(\gamma_{0}{ }^{-1} \nabla_{x} u\right)\right]+\eta_{0} \nabla_{x}^{2} v+\nabla_{x} \sigma_{\mathrm{R}}
$$

where $\nabla_{x}=\partial / \partial x, \rho$ is the mass density, $\mu_{0}$ is the shear modulus, $\gamma_{0}$ is a constant determined by the underlying crystal structure, and $\eta_{0}$ is a vicosity. The period of the strain is given by $\gamma_{\mathrm{p}}=2 \pi \gamma_{0}$. To ensure equilibrium in the absence of applied force, we introduce the random stress $\sigma_{\mathrm{R}}(x, t)$ related to $\eta_{0}$ via the fluctuation-disipation relation $\left\langle\sigma_{\mathrm{R}}(x, t) \sigma_{\mathrm{R}}\left(x^{\prime}, t^{\prime}\right)\right\rangle=2 k_{\mathrm{B}} T \eta_{0} \delta\left(x-x^{\prime}\right) \delta(t-t)$, where $T$ is the temperature. Hereafter space and time will be measured in units of $\ell \equiv \eta_{0} /\left(\rho \mu_{0}\right)^{1 / 2}$ and $\omega_{0}^{-1} \equiv \eta_{0} / \mu_{0}$, respectively. The dimensionless noise strength is given by $\epsilon=k_{\mathrm{B}} T / \mu_{0} \gamma_{0}^{2} \ell$. 
We numerically solve (2) on a $1 \mathrm{D}$ chain $(j=0,1, \cdots, N=600)$ with $\epsilon=0.25$, setting $u(x, t) \rightarrow u_{j}(t)$ and $\partial u / \partial x \rightarrow \gamma_{j} \equiv u_{j+1}-u_{j}$. We apply a constant shear rate $\dot{\gamma}$ at $t=0$ with $u_{0}=0$ and $u_{N} / \gamma_{0}=N \dot{\gamma}$. In Fig.1 the average dimensionless stress $\langle\sigma\rangle(t)=N^{-1} \sum_{j} \sin \left(\gamma_{j}(t) / \gamma_{0}\right)+\dot{\gamma}$ is plotted as a function of the scaled strain $\dot{\gamma} t / \gamma_{\mathrm{p}}$. The system undergoes a pure elastic deformation $\gamma_{j}(t) / \gamma_{0} \cong \dot{\gamma} t$ in the initial stage, but slips (jumps of $\gamma_{j}$ by multiples of $\gamma_{\mathrm{p}}$ ) appear randomly throughout the system with increasing the average strain $\dot{\gamma} t$. The inset shows slips and continuously strained regions. The average slope of the latter regions gives the average stress. For the range of the shear rates in Fig.1, we can see shear-thinning $\sigma_{x y} \propto \dot{\gamma}^{0.7}$ in steady states (if averaged over time).

It is also straighforward to integrate (2) under a fixed shear stress $\sigma_{\text {ext }}$ applied at one end with the other end being pinned. For small $\sigma_{\text {ext }}$ below a yield stress $\sigma_{\mathrm{y}}$, we observe no slip formation, while for $\sigma_{\text {ext }}>\sigma_{\mathrm{y}}$ plastic flow is produced. For the noise strength of $\epsilon=0.25$, we find $\sigma_{\mathrm{y}} \cong 0.2$.

\section{Plastic flow without vacancies}

As a direct generalization of (2), we set up a plastic flow model in two dimensions (2D slip odel). In the continuum limit the strain components are defined by

$$
e_{1}=\nabla_{x} u_{x}+\nabla_{y} u_{y}, \quad e_{2}=\nabla_{x} u_{x}-\nabla_{y} u_{y}, \quad e_{3}=\nabla_{x} u_{y}+\nabla_{y} u_{x}
$$

where $\nabla_{x}=\partial / \partial x$ and $\nabla_{y}=\partial / \partial y$. If we suppose a triangular lattice, the elasticity is isotropic in the harmonic approximation [2], being characterized by the bulk and shear moduli, $K_{0}$ and $\mu_{0}$, but it depends on the orientational angle $\theta$ of one of the crystal axes for large shear deformations. Note that, under rotation of the reference frame by $\theta$, the shear strains $e_{2}$ and $e_{3}$ are changed to $e_{2}^{\prime}$ and $e_{3}^{\prime}$, where [2]

$$
e_{2}^{\prime}=e_{2} \cos 2 \theta+e_{3} \sin 2 \theta, \quad e_{3}^{\prime}=-e_{2} \sin 2 \theta+e_{3} \cos 2 \theta .
$$

We write the elastic energy density in the form, $f_{\mathrm{el}}=K_{0} e_{1}^{2} / 2+\mu_{0} \mathcal{F}\left(e_{3}^{\prime}, e_{2}^{\prime}\right)$. The simplest form of the scaling function $\mathcal{F}$ is of the form

$$
\mathcal{F}\left(e_{3}^{\prime}, e_{2}^{\prime}\right)=\frac{1}{6 \pi^{2}}\left[3-\cos \pi\left(\sqrt{3} e_{3}^{\prime}-e_{2}^{\prime}\right)-\cos \pi\left(\sqrt{3} e_{3}^{\prime}+e_{2}^{\prime}\right)-\cos \left(2 \pi e_{2}^{\prime}\right)\right](5)
$$

and is shown in Fig.2, The $\mathcal{F}$ is invariant with respect to the rotation $\theta \rightarrow \theta+\pi / 3$, is a periodic function of $e_{3}^{\prime}$ with period $\gamma_{\mathrm{p}}=2 / \sqrt{3}$ for $e_{2}^{\prime}=0$ (simple shear deformation), and becomes $\left(e_{2}^{2}+e_{3}^{2}\right) / 2$ for small strains. If we assume $\partial \theta / \partial t=\left(\nabla_{x} v_{y}-\nabla_{y} v_{x}\right) / 2$ for the angle rotation rate, its time integration yields

$$
\theta=\frac{1}{2}\left(\nabla_{x} u_{y}-\nabla_{y} u_{x}\right)+\theta_{0}
$$


where $\theta_{0}$ is independent of $t$ but may depend on $\boldsymbol{r}=(x, y)$. For $\theta=0$ one of the crystal axes is along the $x$ axis. Then the elastic energy $F_{\text {el }}=\int d \boldsymbol{r} f_{\text {el }}$ is a functional of $\boldsymbol{u}$. We assume that the lattice velocity $\boldsymbol{v}=\partial \boldsymbol{u} / \partial t$ obeys

$$
\rho \frac{\partial}{\partial t} \boldsymbol{v}=-\frac{\delta}{\delta \boldsymbol{u}} F_{\mathrm{el}}+\eta_{0} \nabla^{2} v+\nabla \cdot \stackrel{\leftrightarrow}{\sigma}_{\mathrm{R}}
$$

where the first term on the right hand side is also written as $\nabla \cdot \stackrel{\leftrightarrow}{\sigma}$ in term of the elastic stress tensor $\stackrel{\leftrightarrow}{\sigma}$. The symmetric random stress tensor $\stackrel{\leftrightarrow}{\sigma}_{\mathrm{R}}=\left\{\sigma_{\alpha \beta}^{\mathrm{R}}\right\}=\left\{\sigma_{\beta \alpha}^{\mathrm{R}}\right\}$ satisfies $\sigma_{x x}^{\mathrm{R}}+\sigma_{y y}^{\mathrm{R}}=0$, because the bulk viscosity is neglected in (7), and [2]

$$
\left\langle\sigma_{\alpha \beta}^{\mathrm{R}}(\boldsymbol{r}, t) \sigma_{\alpha \beta}^{\mathrm{R}}\left(\boldsymbol{r}^{\prime}, t^{\prime}\right)\right\rangle=2 k_{\mathrm{B}} T \eta_{0} \delta\left(\boldsymbol{r}-\boldsymbol{r}^{\prime}\right) \delta\left(t-t^{\prime}\right) .
$$

We measure space and time in units of $\eta_{0} /\left(\rho \mu_{0}\right)^{1 / 2}$ and $\eta_{0} / \mu_{0}$ and the strains in units of $\gamma_{\mathrm{p}}=2 / \sqrt{3}$. If these scaling units are used, the noise strength $k_{\mathrm{B}} T \eta_{0}$ in $(8)$ is replaced by

$$
\epsilon=k_{\mathrm{B}} T \rho / \gamma_{\mathrm{p}}^{2} \eta_{0}^{2}
$$

We integrate $(7)$ on a $128 \times 128$ square lattice by applying a constant shear rate $\dot{\gamma}$ at $t=0$ with $\epsilon=0.1$. The periodic boundary condition is imposed in the $x$ direction, while $\boldsymbol{u}=\mathbf{0}$ at the bottom $y=0$ and $u_{x}=\dot{\gamma} t$ and $u_{y}=0$ at the top $y=128$. At $t=0$,

the values of $\boldsymbol{v}$ at the lattice sites are Gaussian random numbers with variance $\epsilon^{1 / 2}$ but $\boldsymbol{u}=\mathbf{0}$ and $\theta=0.1$. In Fig. 3 we show the average scaled stress $\left\langle\sigma_{x y}\right\rangle / \mu_{0}$ as a function of the scaled strain $\dot{\gamma} t / \gamma_{\mathrm{p}}$ for $\dot{\gamma}=10^{-3}$ and $10^{-4}$. In the inset we display snapshots of $e_{3}$ and $e_{2}$. Because we start with a perfect crystal with the initial fluctuations only in the velocity, the stress curve drops sharply after the peak with catastrophic formation of slips. Then structurally disordered states are produced where defects are proliferated (strain-induced disordering). Note that slips are accumulations of dislocations [17]. In the initial stage of plastic flow we have simple slips consisting of two dislocations with opposite Burgers vectors $\pm a$ with $a$ being the lattice constant. The elastic energy to create such a slip is minimum in the $x$ and $y$ directions under shear deformation. This is the reason why the slips in Fig. 3 are parallel to the $x$ or $y$ direction. In Fig.4 the shear $\left(=10^{-3}\right)$ is swiched off at (a) $t=190$ before the peak time of the stress, (b) $t=220$ just after the peak time, and (c) $t=440$. The top and bottom boundaries are kept at rest afterwards. Pure elastic deformation is maintained in (a), while no appreciable time evolution is detected after transients in (b) and (c). This means that the structurely disordered states are metastable.

\section{Plastic flow with vacancies}

Usually in the literature, the density deviation $\delta \rho$ is equated with $-\bar{\rho} \nabla \cdot \boldsymbol{u}=-\bar{\rho} e_{1}$ in solids [17], where $\bar{\rho}(\cong \rho)$ is the average density and $|\delta \rho| \ll \bar{\rho}$ is assumeed. In the 
presence of vacancies or interstitials, however, there can be a small difference between these two quantities. Cohen, Flemming, and Gibbs [18] constructed a hydrodynamic description of solids including the new variable $m \equiv \delta \rho / \bar{\rho}+e_{1}$. According to their theory, the vacancy concentration $c$ may be defined by

$$
c=c_{0}-m=c_{0}-\left(\delta \rho / \bar{\rho}+e_{1}\right)
$$

where $c_{0}$ is the average $\langle c\rangle$ dependent on $T$ and $\bar{\rho}$. On the other hand, Granato claimed relevance of interstitials in amorphous solids because they can greatly decrease the shear modulus [20]. However, because vacancies and interstitials are point defects, it may be more appropriate to treat $c$ as the local free-volume fraction $[18,19]$ which can take continuous values at each lattice site. Then $c_{0}$ is the average free-volume fraction and we may assume $0 \leq c_{0} \ll 1$. The role of $m$ or $c$ is expected to be crucial in amorphous solids. We try to include the vacancy variable in our nonlinear slip model (2D vacancy model),

The dynamic equations obeyed by the mass density and the momentum density are of the usual forms,

$$
\begin{aligned}
& \frac{\partial}{\partial t} \delta \rho=-\bar{\rho} \nabla \cdot \boldsymbol{v} \\
& \bar{\rho} \frac{\partial}{\partial t} \boldsymbol{v}=\nabla \cdot \stackrel{\leftrightarrow}{\sigma}+\eta_{0} \nabla^{2} \boldsymbol{v}+\nabla \cdot \stackrel{\leftrightarrow}{\sigma}_{\mathrm{R}}
\end{aligned}
$$

In (12) the force density, the first term on the right hand side, is written in terms of the free energy functional $F=F\{\rho, \boldsymbol{u}\}$ as

$$
\nabla \cdot \stackrel{\leftrightarrow}{\sigma}=-\bar{\rho} \nabla(\delta F / \delta \rho) \boldsymbol{u}-(\delta F / \delta \boldsymbol{u})_{\rho}=-(\delta F / \delta \boldsymbol{u})_{m}
$$

where use has been made of the identities $(\delta / \delta \boldsymbol{u})_{\rho}=(\delta / \delta \boldsymbol{u})_{m}-\nabla(\delta / \delta m) \boldsymbol{u}$ and $(\delta / \delta m) \boldsymbol{u}=\bar{\rho}(\delta / \delta \rho) \boldsymbol{u}$. The random stress tensor $\stackrel{\leftrightarrow}{\mathrm{R}}_{\mathrm{R}}$ satisfies (8). It is important that, in the presence of the vacancy field, the lattice velocity $\partial \boldsymbol{u} / \partial t$ is different from the mass velocity $\boldsymbol{v}$ and is assumed to be of the form,

$$
\frac{\partial}{\partial t} \boldsymbol{u}=\boldsymbol{v}-\lambda_{0}\left(\frac{\delta}{\delta \boldsymbol{u}} F\right)_{\rho}+\boldsymbol{\zeta}_{\mathrm{R}}
$$

where $\lambda_{0}$ is the kinetic coefficient and the components $\zeta_{\alpha}^{\mathrm{R}}$ of the random force vector $\boldsymbol{\zeta}_{\mathrm{R}}$ are characterized by

$$
\left\langle\zeta_{\alpha}^{\mathrm{R}}(\boldsymbol{r}, t) \zeta_{\beta}^{\mathrm{R}}\left(\boldsymbol{r}^{\prime}, t^{\prime}\right)\right\rangle=2 k_{\mathrm{B}} T \lambda_{0} \delta_{\alpha \beta} \delta\left(\boldsymbol{r}-\boldsymbol{r}^{\prime}\right) \delta\left(t-t^{\prime}\right) .
$$

From (12) and (14) the equation for $m$ is expressed as

$$
\frac{\partial}{\partial t} m=\nabla \cdot \lambda_{0}\left[\nabla\left(\frac{\delta}{\delta m} F\right)_{\boldsymbol{u}}+\nabla \cdot \stackrel{\leftrightarrow}{\sigma}\right]+\nabla \cdot \boldsymbol{\zeta}_{\mathrm{R}}
$$


It is worth noting that (16) is similar to the dynamic equation for the concentration in visccoelastic fluid mixtures $[2,21]$. In passing, owing to the special form of (13), the time derivative of the total free energy $F\{\rho, \boldsymbol{u}\}+\int d \boldsymbol{r} \bar{\rho} v^{2} / 2$ becomes nonnegative-definite in the absence of applied stress if the random noises are neglected. This is a self-consistent condition of Langevin equations ensuring attainment of equilibrium [2].

The free energy $F=\int d \boldsymbol{r} f$ is a functional of $\boldsymbol{u}$ and $\delta \rho$ (or $m$ ). We assume the free energy density $f$ in the form,

$$
f=\frac{A}{2} m^{2}+\frac{B}{4} m^{4}+\alpha m e_{1}++\frac{C}{2}|\nabla \delta \rho|^{2}+\frac{K_{0}}{2} e_{1}^{2}+\mu(m) \mathcal{F}\left(e_{3}^{\prime}, e_{2}^{\prime}\right),
$$

where $A, B$, and $C$ are positive constants and $\alpha$ is a coupling constant. The gradient term $(\propto C)$ is introduced to suppress the density fluctuations with short length scales. (We are considering the density fluctuations longer than the peak distance of the pair correlation function.) For simplicity, we neglect the other gradient terms involving the gradients of $m$ and the strains. In our theory it is most important that the shear modulus $\mu(m)$ sensitively depends on $m$. Near glass transitions, with decreasing $m$ or increasing $c, \mu(m)$ is expected to decrease abruptly from a finite value $\mu_{0}$ to zero (= fluid value) around a threshold value of $m$ or $c$.

Analytic calculations of the above model are difficult except for idealized situations. As a simple exercise, let us consider small deviations with wave vector $\boldsymbol{k}$ around a homogeneously strained state with $e_{3}=\gamma$, where the sytem is at rest and the harmonic approximation can be made for the elastic energy. Then the mechanical equilibrium condition allows elimination of $\boldsymbol{u}$ from (16) [2]. We consider the steady-state variance of $\mathrm{m}$ in the long wavelength limit, $\chi_{\mathrm{v}}=\lim _{k \rightarrow 0}\left\langle\left|m_{\boldsymbol{k}}\right|^{2}\right\rangle$, in 2D. In the linear approximation it becomes dependent on the angle of $\boldsymbol{k}$ :

$$
\frac{k_{\mathrm{B}} T}{\chi_{\mathrm{v}}}=A-\frac{\alpha^{2}}{L_{0}}-\frac{2 \alpha}{L_{0}} \mu_{1} \gamma \sin 2 \varphi-\frac{1}{L_{0}}\left(\mu_{1} \gamma\right)^{2}\left[1+(\cos 2 \varphi)^{2} K_{0} / \mu_{0}\right],
$$

where $\sin 2 \varphi=2 k_{x} k_{y} / k^{2}, \mu(m)=\mu_{0}+\mu_{1} m+\cdots$ and $L_{0}=K_{0}+\mu_{0}$. The vacancy diffusion constant is written as $D_{\mathrm{v}}=\lambda_{0} k_{\mid r m B} T / \chi_{\mathrm{v}}$. The steady-state density variance $\chi_{\rho}=\lim _{k \rightarrow 0}\left\langle\left|\rho_{\boldsymbol{k}}\right|^{2}\right\rangle / \rho^{2}$ in $2 \mathrm{D}$ is written as

$$
\chi_{\rho}=\frac{k_{\mathrm{B}} T}{L_{0}}+\left[1+\frac{1}{L_{0}}\left(\alpha+\mu_{1} \gamma \sin 2 \varphi\right)\right]^{2} \chi_{\mathrm{v}} .
$$

The first term is the usual term, while the second term arises from the vacancy fluctuations. The dependence of these quantities on $\gamma$ and the angle $\varphi$ can become appreciable with increasing $\mu_{1}$ and/or decreasing $A$. For $\alpha=0$, for example, the homogeneous state is unstable for $\gamma>\gamma_{\mathrm{c}}=\left(\mu_{0} A\right)^{1 / 2} / \mu_{1}$ against the fluctuations with $\boldsymbol{k}$ along the $x$ or $y$ axis. Note that $\gamma_{\mathrm{c}} \ll 1$ can hold if $\mu_{1} / \mu_{0} \gg 1$. It is easy to confirm that essentially the same result follows in $3 \mathrm{D}$ in both shear and elongational deformations. Thus, in elastically deformed amorphous solids (before onset of plastic 
flow), we predict enhancement of the density fluctuations in particular directions of the wave vector.

We then show our numerical results of our model in (11)-(17) obtained on a $128 \times 128$ square lattice for the case of applying a constant $\dot{\gamma}$. Again the units of space, time, and strains are $\eta_{0} /\left(\rho \mu_{0}\right)^{1 / 2}, \eta_{0} / \mu_{0}$, and $\gamma_{\mathrm{p}}=2 / \sqrt{3}$, respectively. The $m$ is also divided by $\gamma_{\mathrm{p}}$. The dimensionless mass density is given by $\delta \rho / \rho \gamma_{\mathrm{p}}$. Though it is not clear what functional form of $\mu(m)$ is appropriate, we tentatively use

$$
\mu(m)=\mu_{0} \exp \left[-A_{0} /\left(m+m_{0}\right)\right]
$$

for $m+m_{0}>0$ and $\mu(m)=0$ for $m+m_{0} \leq 0$. Here $A_{0}$ is a constant and is set equal to 0.1 in our simulation, while $m_{0}$ is a control parameter representing the closeness to the glass transition. In fact, if $\mu(m)$ goes to zero at $c=c_{\mathrm{c}}$, we have $m_{0}=c_{c}-c_{0}$ from (10). However, essentially the same numerical results follow as long as $\mu(m)$ strongly depends on $m$ (for example, from the simpler form $G(m) \propto\left(m+m_{0}\right)^{2}$ instead of (20)). The other parameter values used are $A=C=1, B=10, \alpha=0, \lambda_{0}=0.1$ and $\epsilon=0.1$. While the initial conditions for $\boldsymbol{v}, \boldsymbol{u}$ and $\theta$ are the same as in the previous case of Figs. 3 and 4, we assign random Gaussian randum numbers with variance 0.1 to $m$. In Fig.5 we show time evolution of the scaled shear stress as a function of the scaled average strain $\dot{\gamma} t / \gamma_{\mathrm{p}}$ at $\dot{\gamma}=10^{-3}$ for $m_{0}=1,0.6$, and 0.2. Fig.6a and Fig.6b display snapshots of $e_{3}, e_{2}, c-c_{0}=-m$, and $\delta \rho$ at $t=250$ and 420 on the curve of $m_{0}=0.6$ in Fig.5. Fig.7 demonstrates that these strain-induced disordered states are metastable as in Fig.4. Salient features are as follows: (i) Plastic flow is induced earlier than in the absence of vacancies, because slip formation is easier in regions with smaller $m$. Onset of plastic flow is sensitive to the initial randomness of $m$. (ii) The effective viscosity $\eta_{\text {eff }}=\left\langle\sigma_{x y}\right\rangle / \dot{\gamma}$ becomes smaller with decreasing the parameter $m_{0}$ in (17) or increasing the elastic inhomgeneity. (iii) The $\eta_{\text {eff }}$ also decreases as a function of time on long time scales because of slow accumulation of vacancies around slips. In our model, though this tendency is considerably suppressed by the quartic term $(\propto B)$ in (16), vacancy accumulation can lead to fluidization at long times after percolation of the slips. (iii) The density fluctuations are induced and are frozen after cessation of shear. As demonstared in Fig.7, the fluctuation variances of the vacancy and the density are

much enhanced than in the initial state. Owing to the gradient term in (16), the density smoothly varies in space (compared with $m$ ).

\section{Concluding remarks}

We have presented a first time-dependent Ginzburg-Landau theory accounting for inhomogeneous nonlinear elastic deformations. It is a coarse-grained theory and the sharp peak of the structure factor, which is essential in the mode coupling theory [22], 
does not come into play. Instead, periodicity of the elastic energy with respect to the strains $e_{3}^{\prime}$ and $e_{2}^{\prime}$ and vacancy-dependence of the shear modulus are two major ingredients of our theory, which bring about metastable structural disordered states with heterogeneities in the vacancy and mass densities. As a closely related effect, Fischer [23] observed excess scattering from nearly static density fluctuations with sizes in a range of 20-200 $\mathrm{nm}$ in glass-forming fluids. We believe that such large-scale frozen fluctuations can arise only as a result of nonlinear elastic deformations induced by structural disorder. We also mention previous simulations. Argon et al. [12] applied a tensile (elongational) strain to a $2 \mathrm{D}$ model to produce slips and shear bands making angles of $\pm 45^{\circ}$ with respect to the stretched direction in agreement with experiments. The same results also follow from our model and will be reported shortly. We will show that the elastic energy to create a slip under tension is minimum for these directions. Ikeda et al. [14] applied a tensile strain to a 3D model to induce a change from a perfect crystal to an amorphous solid.

However, we admit that our results are still preliminary and it remains unclear how our theory corresponds to real physical effects. In particular, the initial conditions ( $\boldsymbol{u}=\mathbf{0}$ and $\theta=$ const.) should be inappropriate for amorphous solids (where structural disorder should preexist even before applying strain). In future work, we should start with initial states with various amounts of disorder and should also examine what parameter values are appropriate for amorphous solids.

We mention analogous and instructive examples. In solids undergoing phase separation or structural transitions, the dependence of the shear modulus on the order parameter, written as $m$, in the form $\mu=\mu_{0}+\mu_{1} m$ is of crucial importance in phase ordering $[2,24]$. In all these cases, local minimization of the nonlinear elastic energy $\left((\delta F / \delta \boldsymbol{u})_{m}=\mathbf{0}\right)$ gives rise to heterogeneous metastable states where time-evolution is pinned. In viscoelastic polymer systems, the composition-dependence of the viscoelastic properties can give rise to shear-induced phase separation $[2,21]$, where the scattered light intensity takes a form similar to $\chi_{\mathrm{v}}$ in (18).

\section{References}

[1] Yamamoto R and Onuki A 1997 Europhys. Lett. 4061

Yamamoto R and Onuki A 1998 Phys. Rev. E 583515

[2] Onuki A Phase Transition Dynamics, (Cambridge University Press, Cambridge, 2002).

[3] Liu A J and Nagel S R (eds.) Jamming and Rheology, (Taylor \& Francis, London and New York, 2001).

[4] J.H. Simmons, R.K. Mohr and C.J. Montrose, J. Appl. Phys. 53, 4075 (1982)

Simmons J H, Ochoa R, Simmons K D and Mills J J 1988 J. Non-Cryst. Solids 105313

[5] Chen H S and Goldstein M 1971 J. Appl. Phys. 431642

[6] Spaepen F, 1977 Acta Metall. 25407 
Argon A S 1979 Acta Metall. 2747

[7] Maeda K and Takeuchi S 1981 Phil. Mag. A 44643

[8] Muranaka T and Hiwatari Y 1995 Phys. Rev. E 51 R2735

[9] Perera D N and Harrowell P 1996 Phys. Rev. E 541652

[10] Yamamoto R and Onuki A 1997 J. Phys. Soc. Jpn. 662545

[11] Kob W, Donati C, Plimton S J, Poole P H and Glotzer S C 1997 Phys. Rev. Lett. 792827

[12] Mott P H, Argon A S and Suter U W 1993 Philos. Mag. A 67931

Bulatov V V, Argon A S 1994 Modelling Simul. Mater. Sci. Eng. 2, 167, 185, 203

[13] Falk M L and Langer J S 1998 Phys. Rev. E 67192

[14] Ikeda H, Qi Y, Çagin T, Samwer K, Johnson W L and Goddard III W A 1999 Phys. Rev. Lett. 822900

[15] Doi M, Harden J L and Ohta T 1993 Macromolecules 264935

[16] Sollich P, Lequeux F, Hébrand P and Cates M 1997 Phys. Rev. Lett. 78, 2020

Sollich P 1998 Phys. Rev. E 58738

[17] Landau L D and Lifshitz E M 1973 Theory of Elasticity (Pergamon, New York)

[18] Flemming P D III and Cohen C 1976 Phys. Rev. B 13500

Cohen C, Flemming P D III and Gibbs J H 1976 Phys. Rev. B 13866

[19] Turnbull D and Cohen M H 1970 J. Chem. Phys. 523038

[20] Granato A V 1996 J de Physique IV (C8) 61

[21] Helfand E and Fredrickson H 1989 Phys. Rev. Lett. 622468

[22] Bengtzelius U, Götze W and Sjölander A 1984 J. Phys. C 175915

Leutheusser E 1984 Phys. Rev. A 292765

[23] Fischer E W 1993 Physica A 201183

[24] Onuki A and Furukawa A 2001 Phys. Rev. Lett. 86452

Onuki A 1999 J. Phys. Soc. Jpn. 685

\section{Figure Captions}

Fig.1, Stress vs strain in units of $\mu_{0} \gamma_{0}$ and $\gamma_{\mathrm{p}}$ obtained from the discretized version of the 1D model (1) after application of constant shear rate. Here $\dot{\gamma} / \omega_{0}=10^{-3}, 10^{-4}, 10^{-5}$, and $10^{-6}$ from above. The inset displays a snapshot of the deviations $u_{j} / \gamma_{0}-\dot{\gamma} t j$ $(0 \leq j \leq 600)$ at $t=720$ for $\dot{\gamma}=10^{-3}$.

Fig.2, Function $\mathcal{F}\left(e_{3}, e_{2}\right)$ defined by (5), which represents the 2D shear deformation energy density divided by $\mu_{0}$ for $\theta=0$.

Fig.3, Stress vs strain for $\dot{\gamma}=10^{-3}$ and $10^{-4}$ obtained from the 2D slip model (7). In the inset $e_{3}$ and $e_{2}$ at $t=400$ for $\dot{\gamma}=10^{-3}$ are shown.

Fig.4, Scaled stress and elastic energy vs time, which demonstates freezing of straininduced diordered states of (7) at long times. The shear is switched off at (a) $t=190$, 
(b) $t=220$, and (c) $t=440$, as indicated by arrows, using the same parameters as for the curve of $\dot{\gamma}=10^{-3}$ in Fig. 3 .

Fig.5, Stress vs strain for $\dot{\gamma}=10^{-3}$ obtained from integration of (11), (12), and (14) using (16) and (17) at $\dot{\gamma}=10^{-3}$. Here $m_{0}=1,0.6$, and 0.2 from above.

Fig.6, Snapshots of $e_{3}, e_{2}, c-c_{0}=-m$, and $\delta \rho$ at (a) $t=250$ and (b) 420 for $\dot{\gamma}=10^{-3}$ on the curve of $m_{0}=0.6$ in Fig. 5 .

Fig.7, Freezing of strain-induced diordered states of the vacancy model. Shown are the density variance $\left[\left\langle(\delta \rho)^{2}\right\rangle\right]^{1 / 2} / \rho \gamma_{p}$, the vacancy variance $\left[\left\langle(\delta m)^{2}\right\rangle\right]^{1 / 2} / \gamma_{p}$, and the average elastic energy $\left\langle f_{\text {el }}\right\rangle$. As in Fig. 4 , the shear rate is switched off at $t=220$. The frozen density profile at $t=2 \times 10^{4}$ is shown in the inset. 


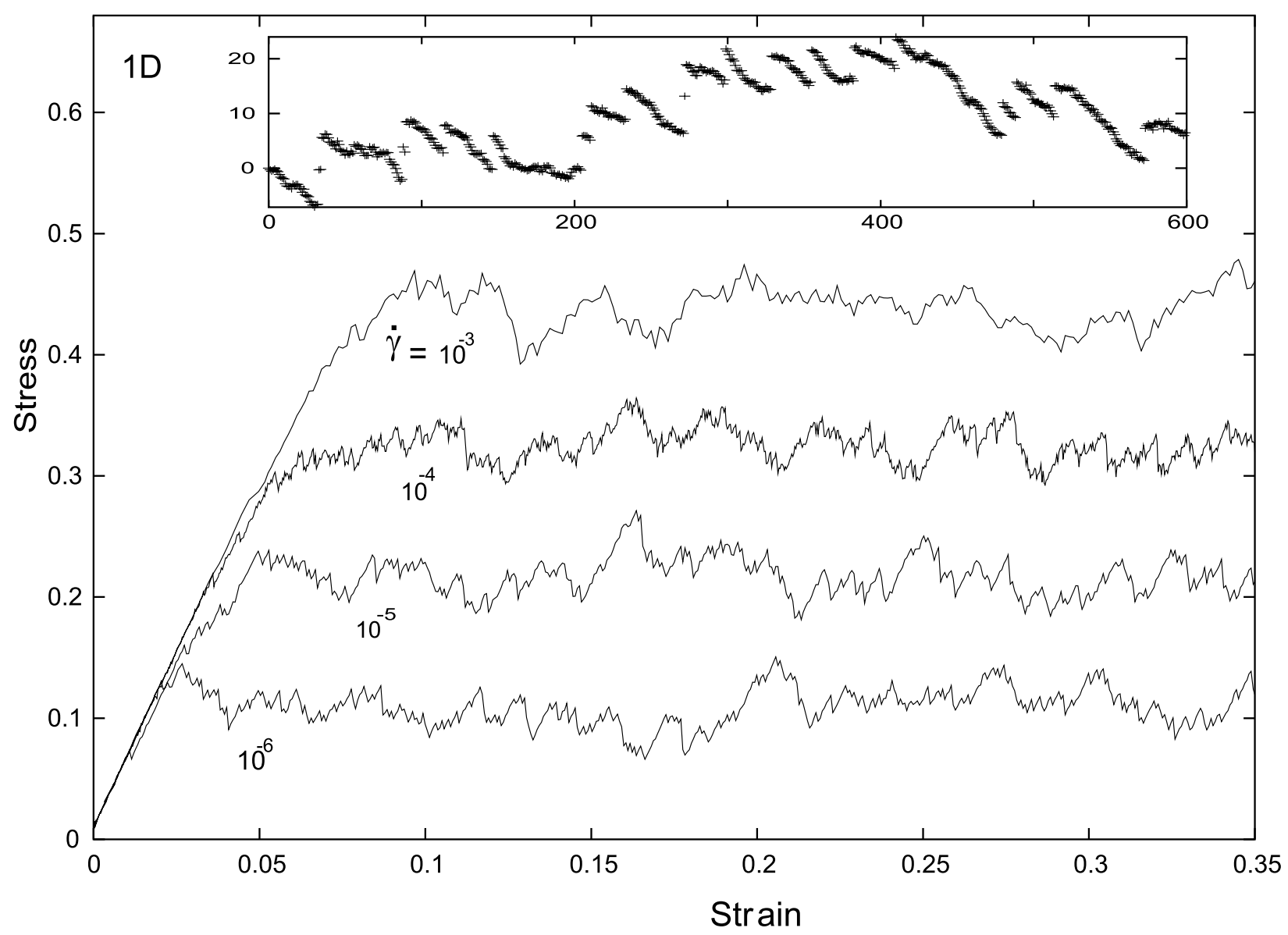




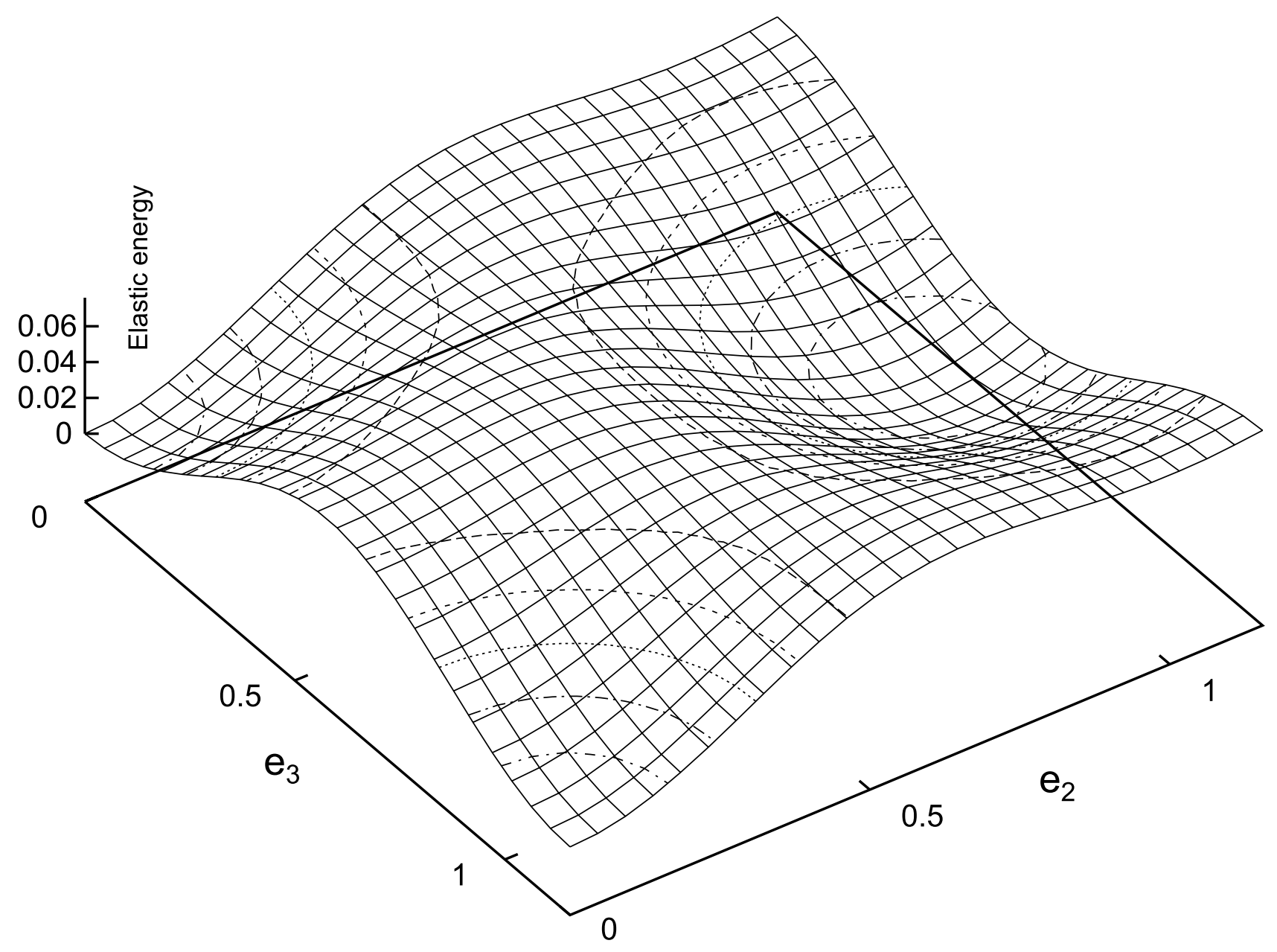




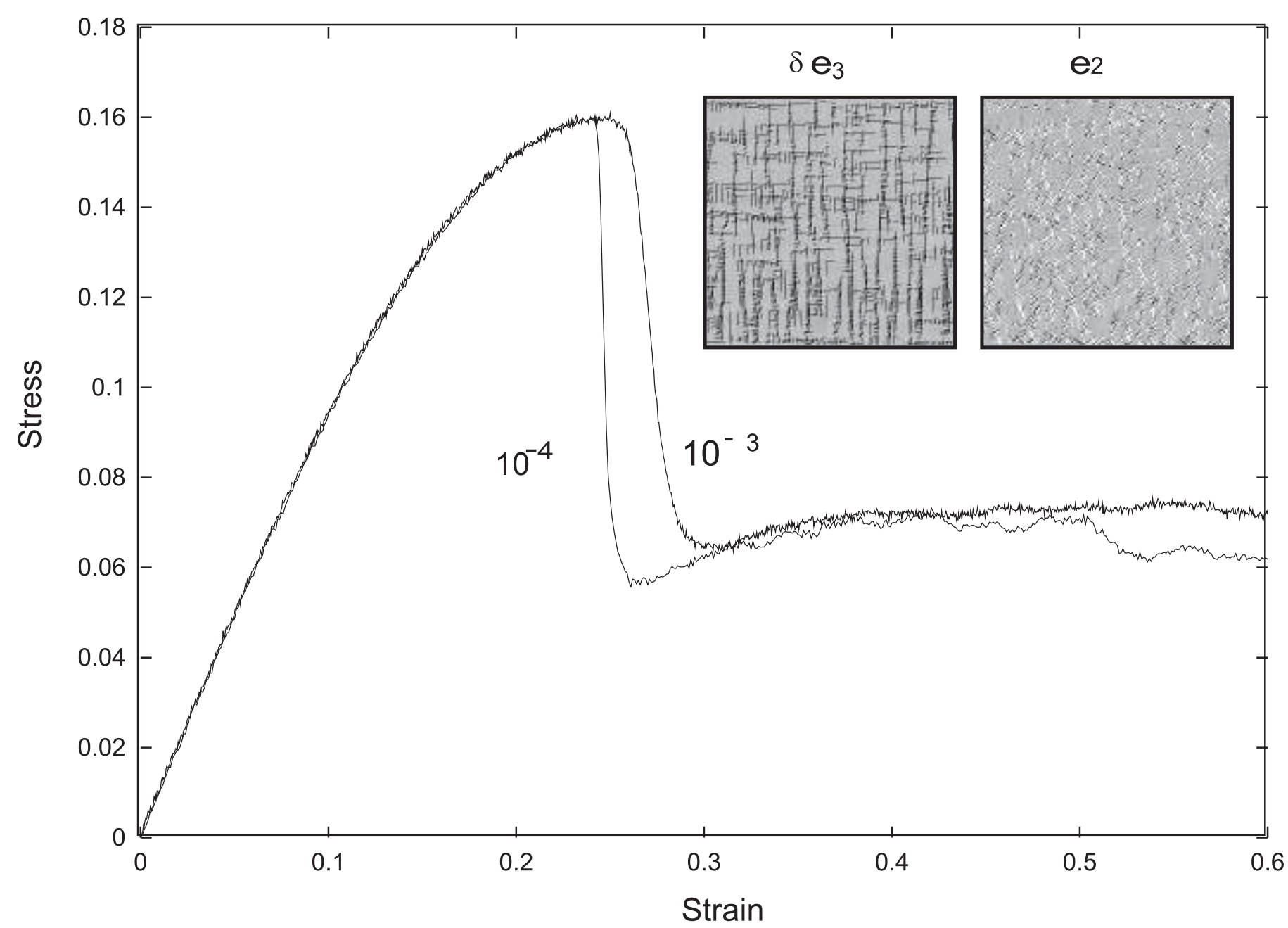




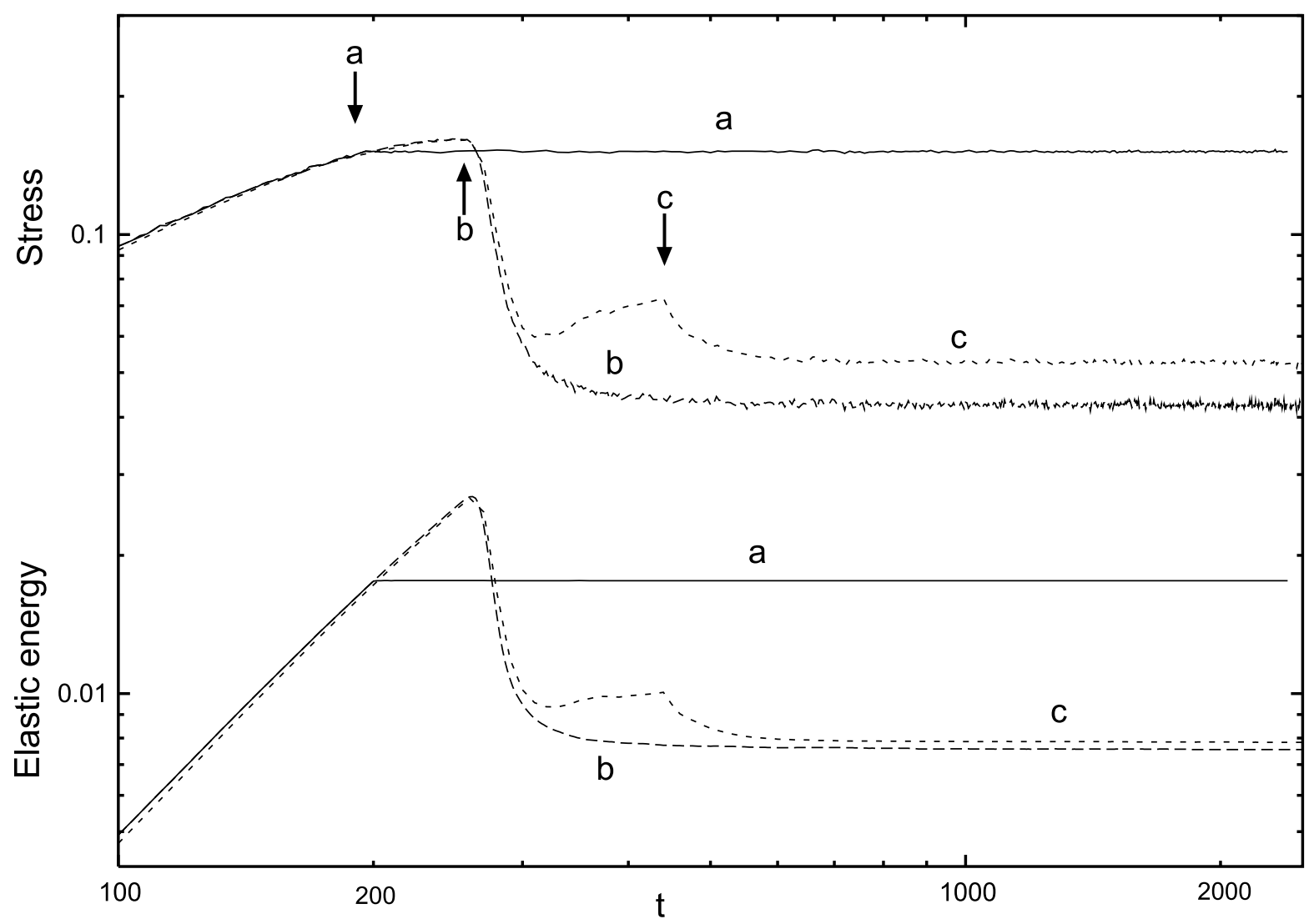




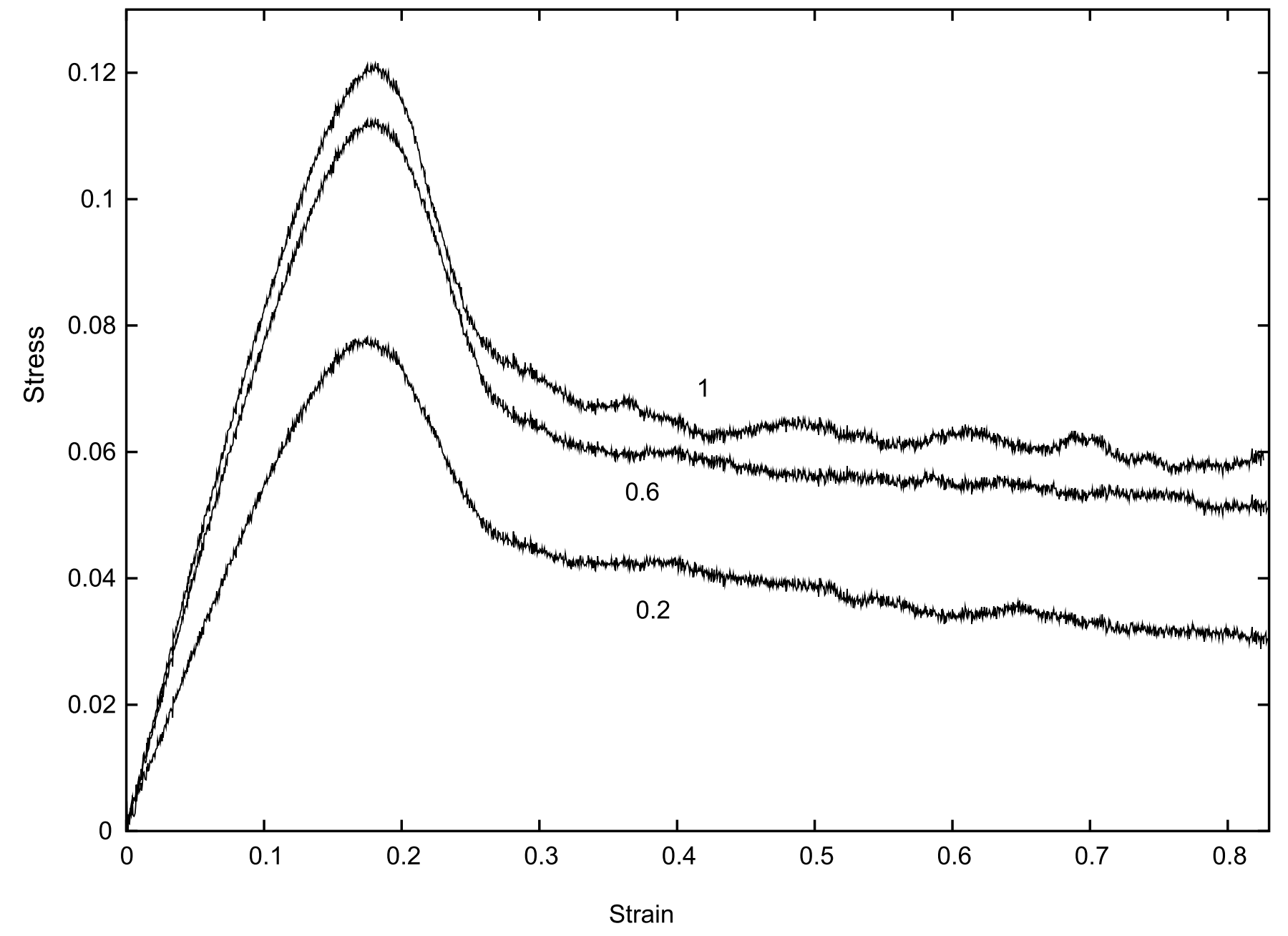


(a) $t=220$
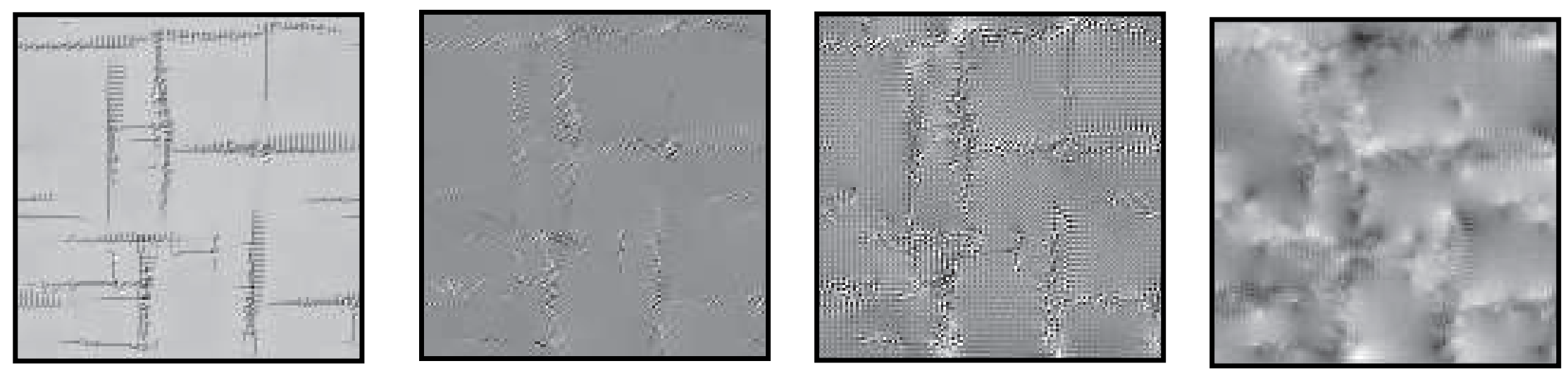

(b) $t=420$
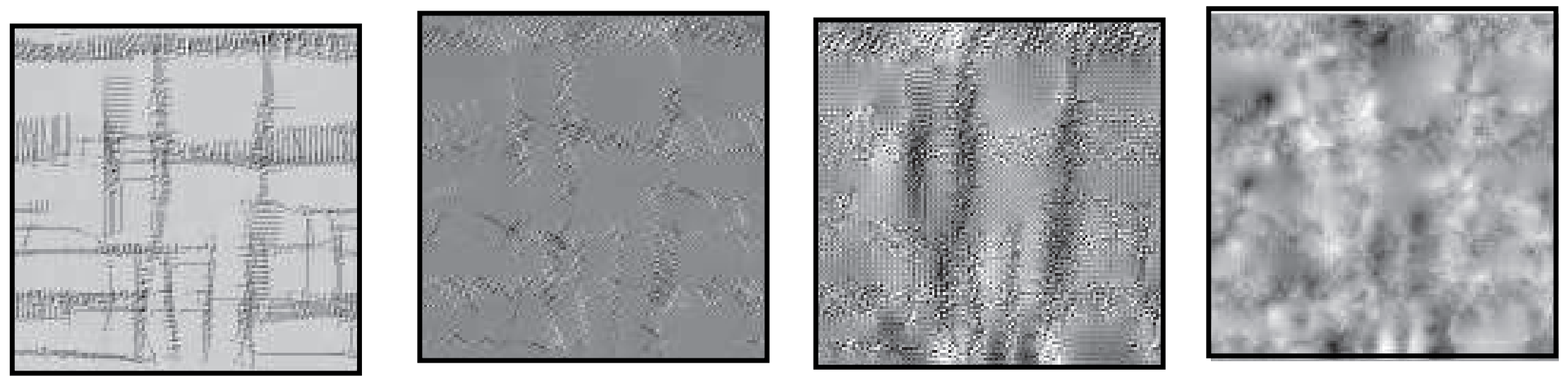

$\delta \mathrm{e}_{3}$

$\mathrm{e}_{2}$

vacancy density

mass density 


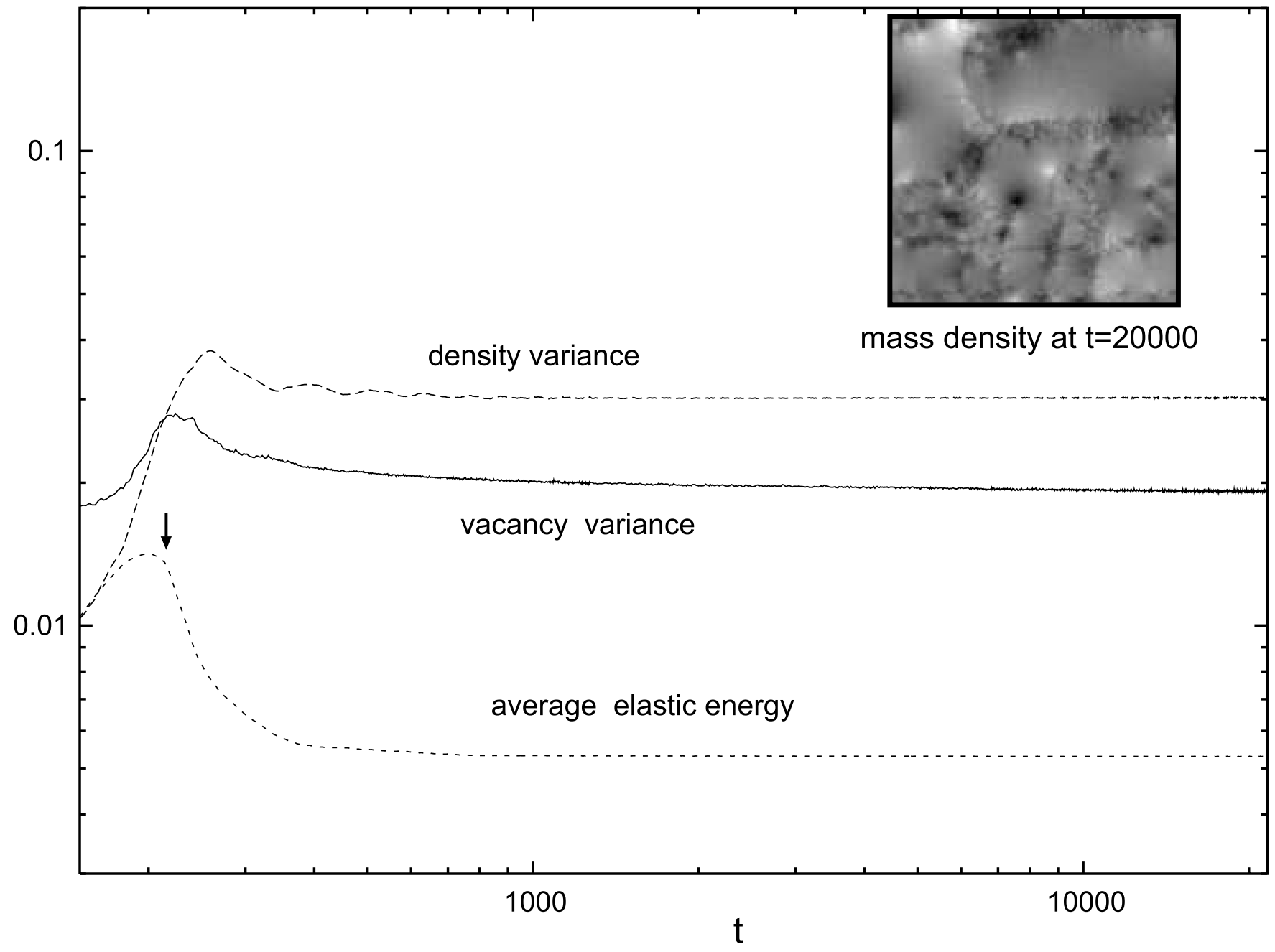

